BENTHAM OPEN
CrossMark
Content list available at: www.benthamopen.com/TORMJ/
DOI: $10.2174 / 1874306401610010105$

RESEARCH ARTICLE

\title{
Effect of Ivacaftor on Objective and Subjective Measures of Cough in Patients with Cystic Fibrosis
}

\author{
Shoaib Faruqi*, Dejene Shiferaw and Alyn H. Morice \\ Department of Respiratory Medicine, Castle Hill Hospital, Centre for Cardiovascular and Metabolic Research, Hull \\ York Medical School, University of Hull, Yorkshire, UK
}

Received: November 10, 2016

Revised: November 14, 2016

Accepted: November 14, 2016

Backstract:

Cough is a major symptom in cystic fibrosis. Ivacaftor is a novel drug which targets the G551D mutation and has been demonstrated to improve lung function and weight in the long term. It also improves symptoms of extra-oesophageal reflux. We wanted to evaluate the effect of ivacaftor on cough in cystic fibrosis.

\section{Methods:}

In two patients with cystic fibrosis the Hull Airway Reflux Questionnaire (HARQ) was completed and objective cough counts were measured prior to and within 4 weeks after initiation of treatment with ivacaftor. Spirometry was also undertaken and weight checked at these time frames.

\section{Results:}

In the first patient the HARQ score decreased from 29 to 11 and objective cough counts from 29 to 9 cough events per hour. Similarly in the second patient the HARQ score decreased from 13 to 9 and objective cough count from 76 to 5 cough events per hour. There was no significant change in spirometric parameters or weight.

\section{Conclusion:}

We have observed early subjective and objective improvement in cough measures on treatment with ivacaftor. We suggest that this improvement could be attributed to improvement of gastro-intestinal function and that cough metrics could be used as early and accurate end points of drug efficacy.

Keywords: Cystic fibrosis, Cough, Ivacaftor, Gastro-oesophageal reflux, G551D mutation, Lung function.

\section{INTRODUCTION}

Cough is one of the predominant symptoms in cystic fibrosis. Patients frequently describe a dry, non-productive background cough with "infective" exacerbations. Ivacaftor is a novel cystic fibrosis transmembrane conductance regulator (CFTR) potentiator targeting the G551D mutation. It has been shown to increase weight and improve lung function in a randomised study [1]; however these parameters are unsuitable for short term efficacy assessments. Objective and subjective measures of cough could potentially act as a rapidly responsive, clinically relevant endpoint for establishing the effectiveness of this class of drugs. We observed the objective and subjective cough response in two cystic fibrosis patients with G551D mutation, following initiation of ivacaftor.

\footnotetext{
* Address correspondence to this author at the Department of Respiratory Medicine, Castle Hill Hospital, Centre for Cardiovascular and Metabolic Research, Hull York Medical School, University of Hull, Yorkshire, United Kingdom; Fax: 014826240681; Email: shoaib.faruqi@hey.nhs.uk
} 


\section{CASE 1}

A 55 year old man with cystic fibrosis complained of prominent cough triggered by lying down, bending over, speaking and on rising from the bed. He suffered bloating, erratic bowel habit and mild dyspepsia. He previously had a Nissen fundoplication for airways reflux. His baseline FEV1 was 0.85 litres (25\% predicted), weight $68.9 \mathrm{~kg}$ and Hull Airway Reflux Questionnaire (HARQ) score of 29 (the upper limit of normal is <13) [2]. Baseline cough monitoring with the semi-automated Hull Automated Cough Counter HACC [3, 4] demonstrated an average of 29 cough events per hour. Within 2 weeks of starting treatment with Ivacaftor he felt significant subjective improvement in his cough. This was reflected in the repeat HACC measurement of 9 coughs per hour (Fig. 1). The HARQ score concomitantly decreased to 11 . FEV1 and weight were unchanged.

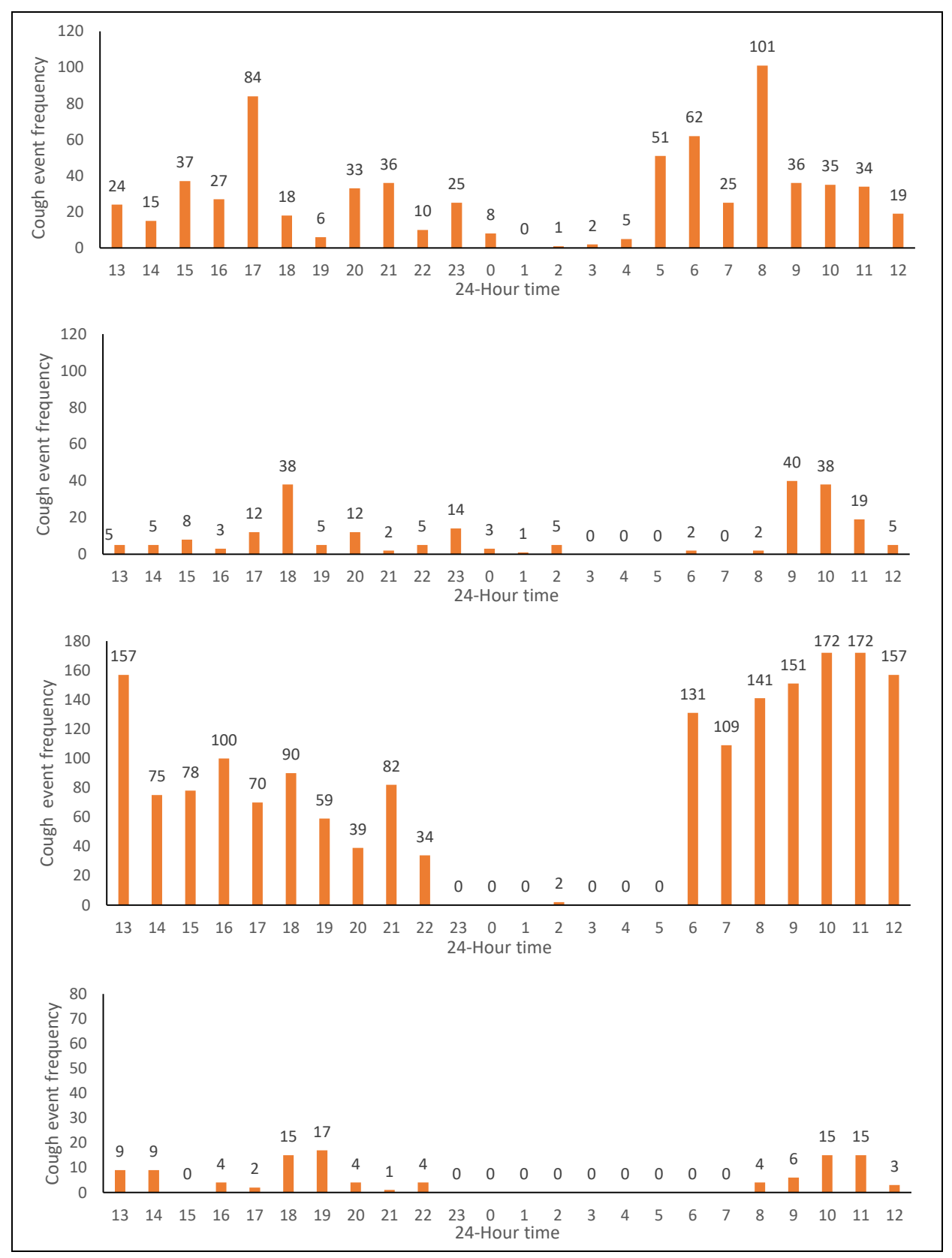

Fig. (1). Twenty four hour cough recordings for both the patients before and after treatment with ivacaftor are depicted. Numbers of coughs recorded in an hour are shown on the " $y$ " axis and the "x" axis demonstrates time in a 24 hour format. The top most panel is a cough recording for the first patient before treatment and the panel below that is on treatment with ivacaftor. The subsequent two panels similarly show the 24 hour cough recording for the second patient prior to ( $3^{\text {rd }}$ panel from top) and on treatment (the last panel) with ivacaftor. 


\section{CASE 2}

An unrelated 35 year old man with cystic fibrosis had similar prominent cough despite Nissen fundoplication. He had baseline FEV1 of 4.64 litres (112\% predicted), weight of $63.8 \mathrm{~kg}$, HARQ score of 13 and 76 cough events per hour were observed on cough monitoring. He spontaneously reported a subjective improvement in cough and his cough monitoring improved to 5 cough events per hour (Fig. 1). The subjective HARQ score was 9 after one month therapy with ivacaftor. Again there was no spirometric or weight change at his follow up visit.

\section{DISCUSSION}

Cystic fibrosis is a multi-system progressive disease with prominent pulmonary manifestations. With progressive pulmonary disease cough is a symptom which is reported by virtually all patients. There are several known pulmonary causes of cough in this patient group such as bronchiectasis, bacterial colonisation and airway inflammation. Gastrooesophageal reflux (GER) is a common cause of chronic cough in the general population. High prevalence of GER and other non-acid symptoms of extra-oesophageal reflux have been reported in the CF population [5]. It has also been reported that surgical treatment of reflux leads to improvement of cough in this patient group, suggesting airway reflux to be an important cause for cough [6].

The HARQ is a self-administered 14-point questionnaire and is a validated tool for the diagnosis and quantification of the symptoms of airway reflux. In our validation study in chronic cough the upper limit of normal was a score of 13 and both our subjects had baseline values demonstrating significant abnormalities. Airway reflux consists of both acid and non-acid aerosolised mist which when deposited in the upper airway may lead to afferent neuronal hypersensitivity, recently termed the Cough Hypersensitivity Syndrome.

The objective assessment of chronic cough has been enhanced by the development of ambulatory cough monitoring systems and this form of assessment may represent the best global objective synthesis of cough, compared to other forms of objective and subjective assessment of cough [7]. Twenty-four hour cough monitoring of both our subjects was well above normal at baseline. We show a very clear and early improvement in cough counts after starting ivacaftor despite no change in weight or spirometry. Hence, cough measurement could be used as an early, precise and clinically relevant endpoint in future drugs development. It may also prove useful as a monitor of compliance for these expensive new agents.

Ivacaftor has a marked positive impact in those with the G551D mutation with sustained improvements in lung function and particularly weight gain. Apart from the formal clinical trial itself, data gained from post approval use of ivacaftor also confirm this effect. Ivacaftor, by universally improving CFTR function, will act on the gastrointestinal tract as is evidenced by weight gain in long term therapy. Zybel et al. have recently reported the novel finding that treatment with ivacaftor leads to sustained and early improvement in symptoms of extra-oesophageal reflux using a panel of validated questionnaires including the HARQ [8]. This symptomatic improvement occurred concurrently with a long-term improvement in weight and lung function. We suggest that the improvement in cough metrics seen in our patients reflects a global improvement of GI function in cystic fibrosis with disease modifying therapy.

\section{CONFLICT OF INTEREST}

The authors confirm that this article content has no conflict of interest.

\section{ACKNOWLEDGEMENTS}

Declared none.

\section{REFERENCES}

[1] Ramsey BW, Davies J, McElvaney NG, et al. A CFTR potentiator in patients with cystic fibrosis and the G551D mutation. N Engl J Med 2011; 365(18): 1663-72.

[http://dx.doi.org/10.1056/NEJMoa1105185] [PMID: 22047557]

[2] Morice AH, Faruqi S, Wright CE, Thompson R, Bland JM. Cough hypersensitivity syndrome: a distinct clinical entity. Lung 2011; 189(1): 73-9.

[http://dx.doi.org/10.1007/s00408-010-9272-1] [PMID: 21240613]

[3] Barry SJ, Dane AD, Morice AH, Walmsley AD. The automatic recognition and counting of cough. Cough $2006 ; 2: 8$. [http://dx.doi.org/10.1186/1745-9974-2-8] [PMID: 17007636]

[4] Crooks MG, Hayman Y, Innes A, Williamson J, Wright CE, Morice AH. Objective measurement of cough frequency during COPD 
exacerbation convalescence. Lung 2016; 194(1): 117-20.

[http://dx.doi.org/10.1007/s00408-015-9782-y] [PMID: 26280405]

[5] Pauwels A, Blondeau K, Mertens V, et al. Gastric emptying and different types of reflux in adult patients with cystic fibrosis. Aliment Pharmacol Ther 2011; 34(7): 799-807.

[http://dx.doi.org/10.1111/j.1365-2036.2011.04786.x] [PMID: 21793864]

[6] Fathi H, Moon T, Donaldson J, Jackson W, Sedman P, Morice AH. Cough in adult cystic fibrosis: diagnosis and response to fundoplication. Cough 2009; 5(1): 1.

[http://dx.doi.org/10.1186/1745-9974-5-1] [PMID: 19149907]

[7] Faruqi S, Thompson R, Wright C, Sheedy W, Morice AH. Quantifying chronic cough: objective versus subjective measurements. Respirology 2011; 16(2): 314-20.

[http://dx.doi.org/10.1111/j.1440-1843.2010.01893.x] [PMID: 21054670]

[8] Zeybel GL, Pearson JP, Krishnan A, et al. Ivacaftor and symptoms of extra-oesophageal reflux in patients with cystic fibrosis and G551D mutation. J Cyst Fibros 2016; 30: pii: S1569-1993-.

C) Faruqi et al.; Licensee Bentham Open

This is an open access article licensed under the terms of the Creative Commons Attribution-Non-Commercial 4.0 International Public License (CC BY-NC 4.0) (https://creativecommons.org/licenses/by-nc/4.0/legalcode), which permits unrestricted, non-commercial use, distribution and reproduction in any medium, provided the work is properly cited. 